\title{
Integración de modelos hidráulicos, de distribución de agua y de cultivo para el análisis holístico de sistemas de riego por aspersión fija
}

\author{
Morcillo García, Manuel ${ }^{1}$, Moreno Hidalgo, Miguel Ángel², Ballesteros González, Rocíoº, Montero Martínez, \\ Jesús $^{4}$, del Castillo Sánchez-Cañamares Amaro ${ }^{5}$
}

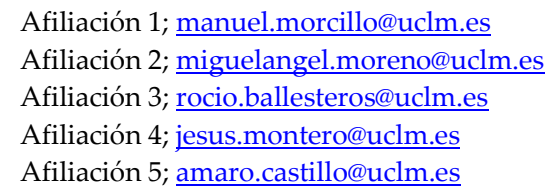

Resumen: En un contexto de recursos limitados, donde, debido al continuo aumento de la población mundial, cada vez hay una mayor demanda de alimentos, lo que se traduce en una mayor explotación de los recursos disponibles, como el agua, que, sumado al elevado consumo de energía para su extracción y aplicación, hacen necesario la modernización de los sistemas de riego y desarrollo de nuevas metodologías y herramientas que permitan gestionar los recursos de una manera más eficiente, obteniendo unas producciones óptimas. En este caso concreto, se ha centrado en los sistemas de riego por aspersión, los cuales se utilizan en cultivos con una gran demanda hídrica y necesitan, además de un correcto diseño y dimensionamiento, una adecuada gestión para el uso eficaz del agua de riego.

Por ello, el objetivo perseguido ha sido la integración de modelos hidráulicos (para evaluar la distribución de presiones y caudales del sistema de riego), de distribución de agua (para determinar la distribución de agua aplicada en el suelo) y de simulación de cultivos (para evaluar el efecto de la distribución de agua sobre la producción del cultivo). Este modelo integrado y holístico permitirá ayudar en el proceso de toma de decisiones sobre el diseño, dimensionado y gestión de sistemas de riego. Para mostrar la potencialidad del modelo integrado se ha aplicado a un caso real, evaluando un diseño realizado por una empresa de riegos privada (utilizando criterios clásicos), llevada a cabo en una parcela de Tarazona de la Mancha (Albacete) con cultivo de cebolla (Allium cepa L.).

La herramienta desarrollada permitirá determinar la producción de cebollas en cada metro cuadrado de la parcela, dada por la distribución de agua en el suelo y una zonificación por las características del mismo, obteniendo los índices; productividad del agua $\left(\mathrm{kg} / \mathrm{m}^{3}\right)$ y productividad de la energía $(\mathrm{kg} / \mathrm{kWh})$, permitiendo determinar la pluviosidad optima del sistema de riego con la que ser más eficientes con los recursos utilizados, pero sin afectar a producción del cultivo. Además, en base a los resultados obtenidos, se puede customizar el sistema de riego de forma localizada, abriéndose un abanico de posibilidades; como mejorar la uniformidad de riego, aplicar más agua en las zonas con mayor potencial productivo, entre otros, en un contexto de riego de precisión. Por lo que se puede concluir que la herramienta desarrollada tiene un elevado potencial, permitiendo ayudar en el proceso de toma de decisiones sobre el diseño, dimensionamiento y gestión de sistemas de riego por aspersión.

Palabras clave: Riego; Aspersión; Precisión; Integración; Modelos. 


\title{
Integration of hydraulic, water distribution and crop models for holistic analysis of fixed sprinkler irrigation systems
}

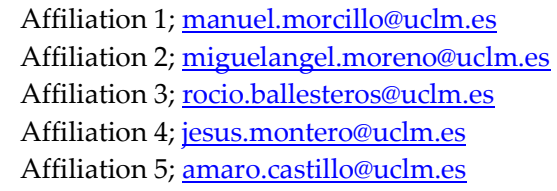

Morcillo García, Manuel ${ }^{1}$, Moreno Hidalgo, Miguel Ángel², Ballesteros González, Rocíoº, Montero Martínez, Jesús ${ }^{4}$, del Castillo Sánchez-Cañamares Amaro ${ }^{5}$

\begin{abstract}
In a context of limited resources, where, due to the continuous increase in the world population, there is an increasing demand for food, which translates into greater exploitation of available resources, such as water, which, added to the high consumption of energy for its extraction and application, make it necessary to modernize irrigation systems and develop new methodologies and tools that allow to manage resources in a more efficient way, obtaining optimal productions. In this specific case, it has focused on sprinkler irrigation systems, which are used in crops with high water demand and require, in addition to proper design and sizing, adequate management for the effective use of irrigation water. Therefore, the objective pursued has been the integration of hydraulic models (to evaluate the distribution of pressures and flows of the irrigation system), water distribution (to determine the distribution of water applied to the soil) and simulation of crops (to assess the effect of water distribution on crop production). This integrated and holistic model will help in the decision-making process on the design, dimensioning and management of irrigation systems. To show the potential of the integrated model, it has been applied to a real case, evaluating a design made by a private irrigation company (using classical criteria), carried out in a plot of Tarazona de la Mancha (Albacete) with onion cultivation. (Allium cepa L.).
\end{abstract}

The developed tool will determine the production of onions in each square meter of the plot, given by the distribution of water in the soil and a zoning by its characteristics, obtaining the indices; water productivity $\left(\mathrm{kg} / \mathrm{m}^{3}\right)$ and energy productivity $(\mathrm{kg} / \mathrm{kWh})$, allowing to determine the optimal rainfall of the irrigation system with which to be more efficient with the resources used, but without affecting crop production. In addition, based on the results obtained, the irrigation system can be customized locally, opening a range of possibilities; how to improve irrigation uniformity, apply more water in areas with greater productive potential, among others, in a context of precision irrigation. Therefore, it can be concluded that the developed tool has a high potential, allowing to help in the decision-making process about the design, dimensioning and management of sprinkler irrigation systems.

Keywords: Irrigation; Sprinkling; Accuracy; Integration; Models. 


\section{Congreso Nacional de Riegos CARTAGENA 2021}

\section{Introducción}

Se espera que la población mundial aumente en 2.000 millones de personas en los próximos 30 años. Para alimentar a esta población cada vez más numerosa, la producción de alimentos deberá aumentar en gran medida, por lo que será necesario producir más con menos. Las proyecciones muestran que, para alimentar una población mundial de 9.700 millones de personas en 2050, sería necesario aumentar la producción de alimentos en un 70\% [1]. La producción de alimentos y el uso del agua están relacionados de forma inseparable. El agua siempre ha sido el principal factor que limita la producción agrícola en gran parte del mundo. Con la competencia cada vez mayor por recursos hídricos no renovables en todo el mundo y la creciente demanda de productos agrícolas, nunca ha sido tan apremiante la necesidad de mejorar la eficiencia y productividad del uso del agua para la producción de cultivos.

El agua de riego es esencial para la producción de cultivos, y cualquier escasez tiene un impacto en los rendimientos finales. Por lo tanto, los agricultores siempre han tendido a regar en exceso (en el caso de cultivos de alto margen bruto), un enfoque que va en contra de la conservación de recursos escasos. En la actualidad, debido a la expansión global de las áreas regables y la disponibilidad limitada de agua de riego, existe la necesidad de optimizar el riego para maximizar en la medida de lo posible el rendimiento de los cultivos [2]. Para dicha optimización en el uso del agua, se demanda una correcta planificación del riego, ya que sólo se requerirá de un evento de riego cuando la cantidad de lluvia sea insuficiente para compensar las pérdidas de agua por evapotranspiración. El objetivo principal del riego es la aplicación del agua en el momento preciso y con la cantidad precisa de agua.

Para mejorar la eficiencia y la productividad del uso del agua para la agricultura, es necesario la modernización de los sistemas de riego, donde los sistemas de riego tradicionales como el riego por superficie o gravedad se vean sustituidos por sistemas más modernos como el riego localizado o por aspersión.

Es cierto que esta modernización también lleva consigo un aumento de la demanda energética, que unido a los aumentos de las tarifas eléctricas hacen que se incrementen en gran medida los costes. Además, la energía utilizada genera importantes emisiones de gases de efecto invernadero (GEI), que contribuyen a acelerar el cambio climático [3]. Por ello, se necesita optimizar las instalaciones de riego, pero no solamente desde el punto de vista productivo, si no también desde un punto de vista de sostenibilidad, por lo que siempre se deben considerar criterios tanto ambientales como económicos. Por tanto, es necesario desarrollar herramientas y modelos que puedan contribuir directamente a mejorar el uso del agua y la energía en el riego, con un enfoque holístico para el diseño y la gestión de la infraestructura del riego [4].

En cuanto a los diferentes sistemas de riego, debido a las características del sistema de riego por aspersión, es el sistema más adecuado para cultivos con una elevada densidad de plantación, como por ejemplo maíz, bulbos, tubérculos, cereales y leguminosas. La idoneidad del riego por aspersión depende de factores de diseño y de funcionamiento. Como efectos derivados de la aplicación de agua mediante sistemas de aspersión se encuentran: la relación entre la velocidad de aplicación (pluviometría del sistema) y la capacidad de infiltración de agua del suelo, produciéndose escorrentía si la primera supera a la segunda, el posible deterioro de la superficie del terreno por el impacto de las gotas si estas son muy grandes, la uniformidad de distribución en superficie y su gran dependencia de la acción del viento, intensidad y dirección [4]. Para minimizar los posibles efectos adversos que pueden ocasionar los sistemas de aspersión, se ha de realizar un diseño y dimensionado óptimo.

Debido a que las redes de distribución de agua en aspersión son sistemas complejos cuyo modo de funcionamiento escapa muchas veces al control de los operadores. El desarrollo no planificado de la red, unido al comportamiento altamente no lineal de sus componentes, obligan hoy en día a utilizar 


\section{Congreso Nacional de Riegos CARTAGENA 2021}

modelos de simulación por ordenador [5]. El programa informático más utilizado para la simulación de redes hidráulicas es EPANET, el cuál es capaz de realizar una simulación de período prolongado del comportamiento hidráulico y de la calidad del agua dentro de redes de tuberías presurizadas [6]. El problema de estos modelos hidráulicos es que se llega a nivel emisor en los modelos más avanzados, pero realmente no se evalúa lo que ocurre cuando el agua sale por los aspersores. Existen programas como SIRIAS (SImulación de RIego por ASpersión), que es capaz de simular la distribución de agua de los aspersores bajo la influencia del viento, utilizando la teoría balística [7], y SORA (SOlapamiento en Riego por Aspersión), que muestra la superposición para cualquier marco de riego [7]. El software SORA sólo solapa 4 aspersores y no tiene en cuenta que estos puedan tener distinta presión. Para su aplicación en una parcela real de riego, se presenta la necesidad de escalar el estudio a nivel sector y que contemple la capacidad de que cada uno de los emisores pueda tener distinta presión. En el caso de los cultivos, existen modelos informáticos que pretenden simular su comportamiento ante diferentes escenarios de manejo. Estas herramientas se han desarrollado con el conocimiento científico de la fisiología de las plantas, la ciencia del suelo, la agroclimatología y su relación con el manejo de los cultivos en campo [8]. Actualmente existen distintos modelos de cultivo, entre los que destacan AquaCrop [9], Cropsyst [9] y Sticks [9], entre otros. El software AquaCrop simula el crecimiento, la productividad y el uso de agua de un cultivo día a día, según se vea afectado por condiciones cambiantes en la disponibilidad de agua y las condiciones ambientales [10].

Actualmente no existen modelos integrales que evalúen el efecto de la distribución de presiones en el sistema de riego y la distribución de agua sobre la producción en cada punto de la parcela, en un contexto de agricultura de precisión. Estos modelos integrados permiten evaluar las decisiones realizadas en el diseño y dimensionado de los sistemas de riego de una forma global, hasta llegar a la producción en cada parte de la parcela, con las consecuencias, económicas asociadas.

Así, el objetivo de este trabajo es integrar modelos hidráulicos, de distribución de agua y de cultivo para el análisis holístico de sistemas de riego por aspersión fija. Para conseguir este objetivo se aplicará la metodología desarrollada a una parcela comercial localizada en el término municipal de Tarazona de La Mancha, Albacete.

\section{Materiales y métodos}

\subsection{El caso de estudio.}

La parcela de estudio se encuentra ubicada en el término municipal de Tarazona de la Mancha (Albacete), tiene una superficie de 2,82 ha y cuenta con un sistema de riego por aspersión en un marco de 17,5 m x 17,5 m, con aspersores circulares UNIRAIN F46 de dos boquillas (5/32"+3/32") con vaina prolongadora del chorro y aspersores sectoriales UNIRAIN P45LP de una boquilla (5/32") con vaina prolongadora del chorro, donde el agua de riego es abastecida por la comunidad de regantes SAT no 8770 "Soreta". La parcela se encuentra a su vez dividida en 2 sectores de riego, el sector 1 tiene una superficie de 1,52 ha y cuenta con 60 aspersores (45 circulares y 15 sectoriales), mientras que el sector 2 tiene una superficie de 1,30 ha y cuenta con 54 aspersores (39 circulares y 15 sectoriales). En cuanto al cultivo establecido en el año de estudio, se trata de cebolla (Allium cepa L.) de siembra, la cual se ha sembrado en camas de $1 \mathrm{~m}$ de ancho, contando cada una con 6 filas y una separación entre bulbos de $15 \mathrm{~cm}$, con un total de 59 camas a lo largo de la parcela.

Por la variabilidad del suelo a lo largo de la parcela, se ha realizado una zonificación, ya que, dependiendo de las características del mismo, el cultivo se desarrollará de manera diferente, afectando de forma distinta la cantidad de agua de riego aplicada. En primer lugar, se ha medido la conductividad eléctrica del suelo mediante una sonda EM38, realizando un total de 1.063 mediciones sobre una malla 


\section{Congreso Nacional de Riegos CARTAGENA 2021}

de $5 \mathrm{~m} \times 5 \mathrm{~m}$ y total de 279 mediciones sobre una malla de $10 \mathrm{~m} \times 10 \mathrm{~m}$. Paralelamente, se han tomado un total de 7 muestras de suelo repartidas por toda parcela, las cuales han sido analizadas en el Instituto Técnico Agrario Provincial de Albacete (ITAP). En base al estudio de la conductividad eléctrica y a las muestras de suelo analizadas se ha dividido la parcela en 4 zonas diferentes.

\subsection{Caracterización y modelización hidráulica del sistema de riego.}

En primer lugar, se ha modelado el sistema de riego en el programa Epanet, donde, para poder ubicar cada uno de los elementos que componen el sistema de riego, se ha georreferenciado cada uno de los aspersores de la parcela con un GPS Leica Zeno 20 (de precisión centimetrica). La ubicación de cada uno de estos puntos se ha llevado a AutoCAD, donde se han añadido el resto de elementos (tuberías y válvulas) siguiendo el plano de instalación del sistema de riego. Con un programa creado en entorno MATLAB, se ha obtenido finalmente el diseño del modelo hidráulico de Epanet para cada uno de los sectores de riego, integrado el diseño del sistema de riego de AutoCAD (indicando los diferentes diámetros de las tuberías en función del color asignado) y el Modelo Digital del Terreno (MDT) con paso de malla de $5 \mathrm{~m}$ descargado del Centro de Descargas del CNIG (Centro Nacional de Información Geográfica), con el objetivo de conocer la cota del suelo y así poder ubicar en altura cada uno de los elementos.

Para determinar el coeficiente de descarga de los aspersores, estos fueron ensayados en la Estación de Seguimiento de Maquinaria Agrícola (ESEMA), donde se realizaron el mismo número de ensayos para los dos tipos de aspersores (circulares y sectoriales), con presiones de $20 \mathrm{mca}, 25 \mathrm{mca}, 30 \mathrm{mca}, 35$ mca, $40 \mathrm{mca}, 45 \mathrm{mca}, 50 \mathrm{mca}$ y $55 \mathrm{mca}$. Realizando un total de 50 ensayos, debido a que se realizaron repeticiones con distintas unidades, con el objetivo de disminuir el posible error (tanto de medida, como de variabilidad entre unidades de un mismo modelo de aspersor), obteniendo para cada una de dichas presiones el caudal emitido, mediante un caudalímetro electromagnético (permitiendo obtener la curva presión-caudal introducida a cada aspersor en el modelo hidraulico de Epanet) y la curva radial de distribución de agua, con pluviómetros de área conocida colocados a 0,6 m de separación.

Se ha realizado una calibración del modelo de Epanet, con el objetivo de introducir al programa las rugosidades optimas en cada tuberia y ajustarlo de una forma precisa al comportamiento real del sistema, para ello se han utilizado transductores de presión colocados estratégicamente en la parcela y conectados a unos Datalogger desarrollados y fabricados en por la sección del Instituto de Desarrollo Regional (IDR) Precisión Agroforestal y Cartográfica (PAFyC) de la Universidad de Castilla-La Mancha (UCLM), permitiendo almacenar datos de presión cada segundo. Para dicha calibración se ha colocado en cada sector y evento de riego, un total de 4 transductores de presión, los cuales se encuentran funcionando de forma simultánea. En el primer sector se ha colocado el primer transductor en cabecera (con el objetivo de almacenar todos los datos de presión inicial), el segundo se ha colocado en el último aspersor del primer ramal (para la calibración del ramal con mayor presión), el tercero se ha colocado en la válvula correspondiente al último ramal (para la calibración de la tubería secundaria) y el cuarto se ha colocado en el último aspersor del ramal donde se encuentra la situado el transductor de la válvula (para la calibración del ramal con menor presión). En cuanto al segundo sector se ha seguido la misma estrategia, pero en este caso se han realizado más determinaciones en otros puntos, moviendo el segundo transductor al segundo y tercer aspersor más desfavorable.

Con los datos obtenidos por los datalogger para cada sector, se ha creado un programa en entorno MATLAB utilizando la dll de Epanet, el cual introduce la presión en cabecera en cada segundo durante todo el riego objetivo y realiza para cada una de estas presiones tantas simulaciones en Epanet como es necesario, modificando en cada una de ellas la rugosidad de tres tipos de tuberías previamente 


\section{Congreso Nacional de Riegos CARTAGENA 2021}

asignadas (tuberías primarias y secundarias, tuberías porta-aspersores y aspersores), de manera que, se comparan (para todas las simulaciones) los resultados de presiones simuladas en los aspersores donde se han colocado los transductores de presión, con el valor real de dichas presiones medido en campo, realizando un proceso de optimización, el cual determina de manera estadística el valor de rugosidad de cada uno de los diferentes elementos integrados que minimiza el error, obtenido así una precisa calibración del modelo hidráulico de Epanet.

La planificación de riegos del cultivo de cebolla la ha llevado a cabo el propio agricultor de la parcela, apoyado por una sonda de humedad colocada por la empresa Nutricontrol, además, dicha empresa ha instalado un programador web de riegos, el cual, ademas de permitir controlar los solenoides que abren y cierran la válvula principal y la de los dos sectores de riego, registra en su plataforma web los valores de caudal y presión en cabecera de cada uno de los eventos de riegos, con intervalos de tiempo de registro de entre 10 y 20 minutos. Estos datos, ampliados con los registros del datalogger instalado en cabecera, se han utilizado para registrar las presiones y caudales de cada uno de los eventos de riego.

\subsection{Determinación de la distribución de agua en la parcela}

Para la simulación de la distribución de agua en el suelo de cada aspersor, se ha utilizado el programa informático SIRIAS, el cual es capaz de simular la distribución de agua bajo la influencia del viento, utilizando teoría balística para simular la trayectoria de las gotas descargadas, de manera que, integrando las variables del viento intensidad y dirección, permite obtener una distribución de agua distorsionada por el viento [11]. Además, el programa tiene en cuenta las perdidas por evaporación y arrastre derivadas del proceso del riego. Para ello, a SIRIAS se le han introducido las curvas radiales ensayadas (tanto de los aspersores circulares como de los sectoriales), realizando simulaciones cada 1 mca, con un rango de presiones desde $25 \mathrm{mca}$ a $55 \mathrm{mca}$, donde para cada una de las presiones se han simulado todas las condiciones de viento posibles, con rangos de intensidades cada $1 \mathrm{~m} / \mathrm{s}$, desde una hipótesis sin viento $(0 \mathrm{~m} / \mathrm{s})$ hasta un máximo de $6 \mathrm{~m} / \mathrm{s}$ y con intervalos de dirección del viento cada $10^{\circ}$, obteniendo una base de datos de 6.727 posibilidades de presión y variabilidad de viento para cada tipo de aspersor (13.454 en total).

Para poder realizar una simulación de la distribución de agua en un sector completo y real de riego, el grupo de investigación PAFyC ha desarrollado el programa SORA 2021, programado en entorno MATLAB, el cual es capaz de realizar una simulación de la distribución del agua de riego en cada metro cuadrado de la parcela, distinguiendo entre sectores de riego, donde, la presión en cabecera es cambiante (en este caso, se ha utilizado para toda la campaña de riegos una media de presiones cada 30 minutos, usando los registros de la plataforma de Nutricontrol y del datalogger propio ubicado en cabecera) y en base a esto, integrado el modelo hidráulico de Epanet (con la dll), cada aspersor tendrá una presión diferente para el período de tiempo de riego simulado, además, el programa integra las variables del viento (intensidad y dirección), utilizando datos climáticos de viento cada 30 minutos (coincidiendo con los tiempos de riego y la media de presiones) obtenidos de la estación de la red SIAR de Motilleja, ubicada a $18 \mathrm{~km}$ de la parcela. Para esta simulación, el programa utiliza la base de datos de simulaciones de SIRIAS, la cual contiene todas las posibilidades de presiones y dirección e intensidad de viento, en este caso se han tratado todos los aspersores del sector como circulares, puesto que los puntos a analizar se encuentran fuera del radio de la superficie mojada de dichos aspersores. De manera que, se ha realizado la simulación del sector 1, obteniendo el agua aplicada durante toda la campaña de riegos en cada metro cuadrado de suelo. Tras dividir el valor obtenido entre el número total de horas de riego, se ha obtenido la pluviosidad. 


\subsection{Monitorización del cultivo y determinación de la producción}

Para el seguimiento del cultivo, se realizaron un total de 5 vuelos de dron, espaciados en el tiempo a lo largo del ciclo de cultivo, portando una cámara RGB (con un tamaño de pixel de $1 \mathrm{~cm}$ ) y 2 cámaras multiespectrales (Micasense RedEdge MX y Parrot Sequoia), aunque en este caso solo se han utilizado las ortoimágenes multibanda de la cámara Micasense Red Edge MX (con un tamaño de pixel de $5 \mathrm{~cm}$ ) con la que se ha determinado el índice de vegetación de diferencia normalizada (NDVI). Antes de realizar los 3 últimos vuelos, se colocaron estratégicamente en cada una de las 4 zonificaciones por tipo de suelo unos marcos metálicos de $1 \mathrm{~m} \times 1 \mathrm{~m}$ (coincidiendo con el ancho de las camas de la cebolla), los cuales se pueden diferenciar en las ortoimágenes, en estos marcos se realizó un muestreo de biomasa, donde se contaron el número de cebollas y se cogió una muestra de 5 bulbos (con el calibre más representativo del marco), las cuales fueron pesadas en fresco y posteriormente secadas en una estufa para obtener la materia seca.

Para poder comparar la producción del cultivo en cada uno de los cuatro puntos con el resto de parámetros, solamente se tuvo en cuenta el peso en fresco de las 5 cebollas muestreadas el día 13/08/2021 en cada una de las zonas de la parcela, donde el bulbo ya había alcanzado su máximo desarrollo y todavía el cuello de la cebolla se encontraba turgente.

Con 3 de los 4 marcos de $1 \mathrm{~m} \times 1 \mathrm{~m}$ (correspondientes a las 3 zonas por tipo de suelo que se encuentran en el sector 1) se ha realizado una comparación entre el NDVI (obtenido con la cámara multiespectral Micasense RedEdge MX el día 13/08/2021, coincidiendo con la realización del muestreo), la producción de cebollas obtenida en el muestro y la pluviosidad media en ese metro cuadrado concreto, con el objetivo de encontrar una relación entre la pluviosidad, el índice NDVI y la producción.

\section{Resultados y discusión}

Se ha obtenido la distribución de agua en el suelo mediante el programa SORA 2021, pero solo se ha tenido en cuenta en el sector 1, siendo suficiente para comprobar la influencia del riego en el desarrollo del cultivo, ya que, el principal objetivo para este trabajo es comprobar de forma visual que la pluviosidad de las diferentes zonas se ajusta al índice NDVI (Figura 1) y relacionar la pluviosidad, el NDVI y la producción (utilizando el peso de las muestras recogidas), en los marcos de $1 \mathrm{~m} \times 1 \mathrm{~m}$ donde se realizaron los muestreos de biomasa (Figuras 2, 3 y 4). 


\section{AREYD}

CARTAGENA 2021

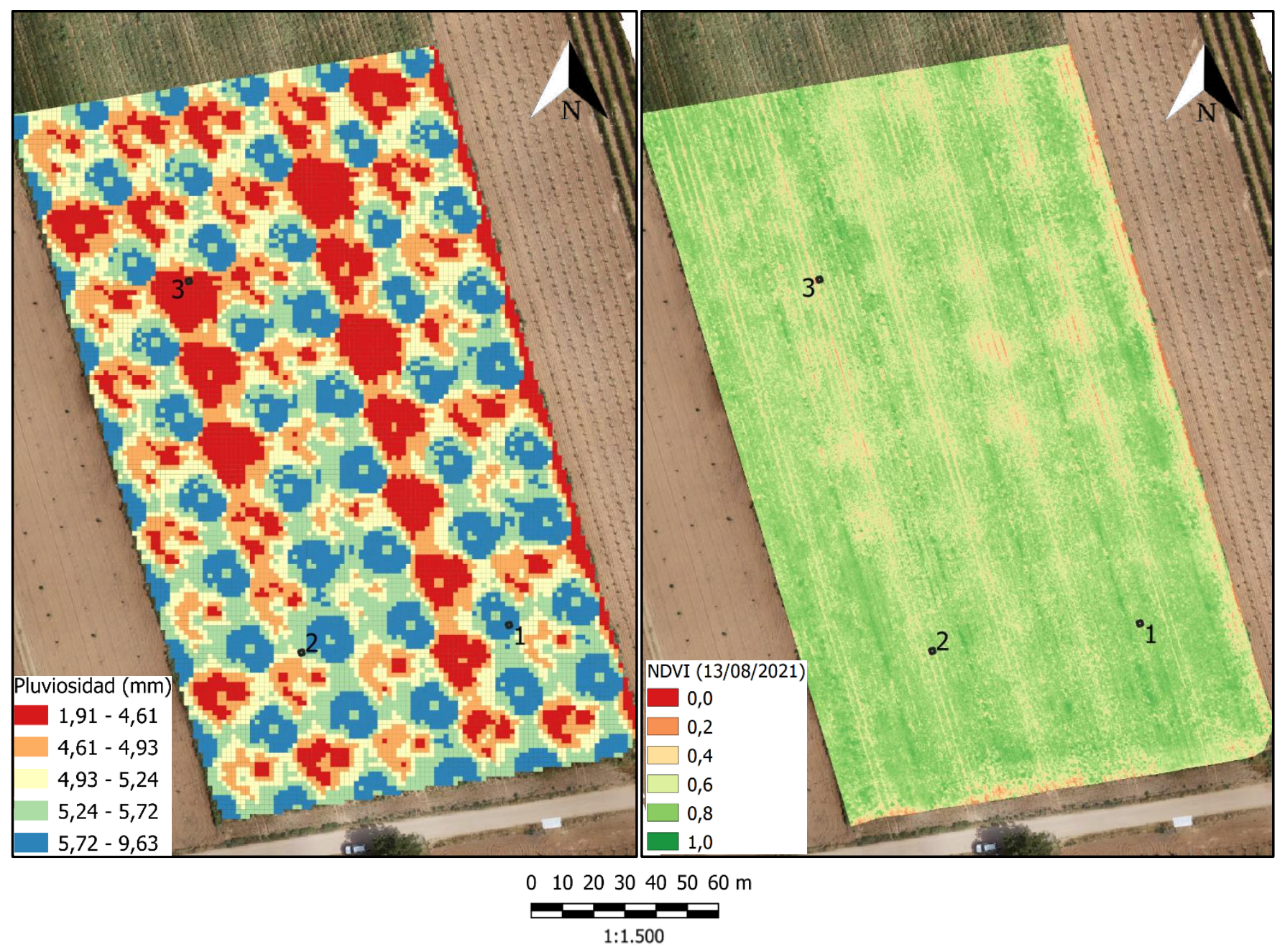

Figura 1. Pluviosidad del sistema de riego (izquierda) e índice NDVI el día 13/08/2021 (izquierda). Los puntos muestran las zonas de control de producción.

En la figura 1 se puede observar de forma visual que las zonas con menor y mayor pluviosidad coinciden directamente con las zonas de menor y mayor índice NDVI, incluso sin tener en cuenta la zonificación por características de suelo. En cuanto al mapa de pluviosidad, para la comparación con el índice NDVI hay que descartar el área de influencia de la primera línea de aspersores del lateral izquierdo y de la zona inferior de la parcela, debido a que los aspersores de esas dos líneas son sectoriales y la simulación se ha realizado teniendo en cuenta todos los aspersores como circulares (por lo que en dicha zona la cantidad de agua aplicada es mayor). En posteriores actualizaciones del programa SORA 2021 se introducirá la base de datos SIRIAS de los aspersores sectoriales, siendo el programa capaz de distinguirlos y utilizar la curva radial correspondiente. También se añadirá el sector 2 de riego, con el objetivo de analizar toda la parcela. 


\section{Congreso Nacional de Riegos}

CARTAGENA 2021

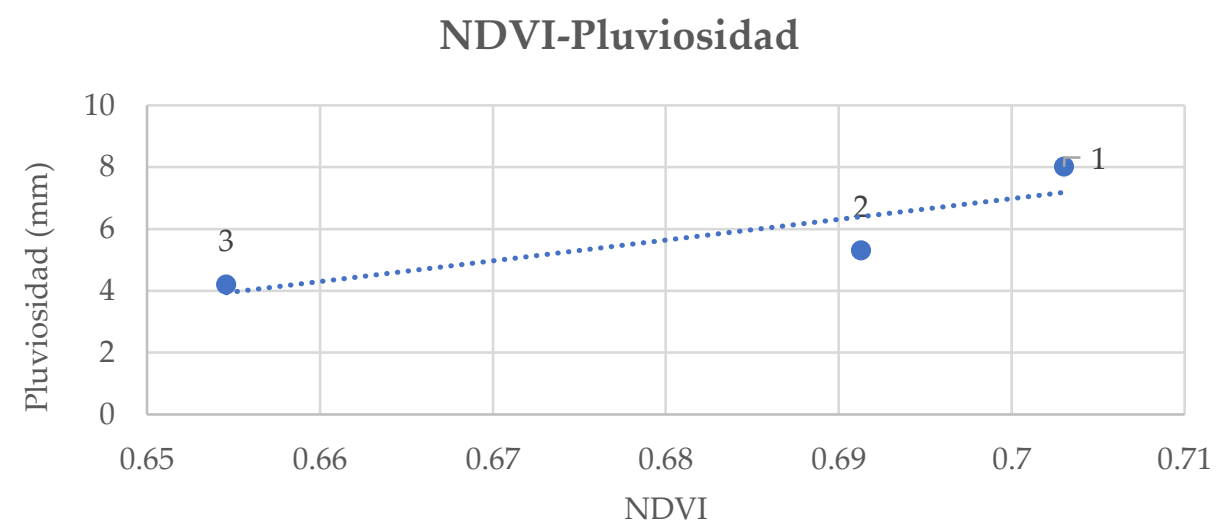

Figura 2. Relación entre el índice NDVI y la pluviosidad en cada uno de los marcos de muestreo.

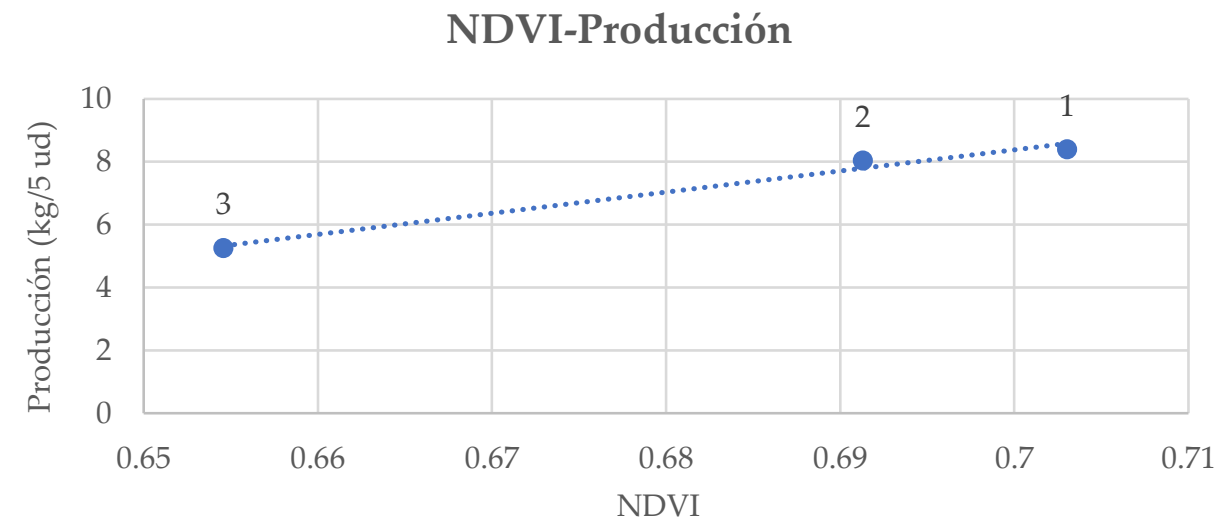

Figura 3. Relación entre el índice NDVI y la producción obtenida en cada uno de los marcos de muestreo.

\section{Pluviosidad-Producción}

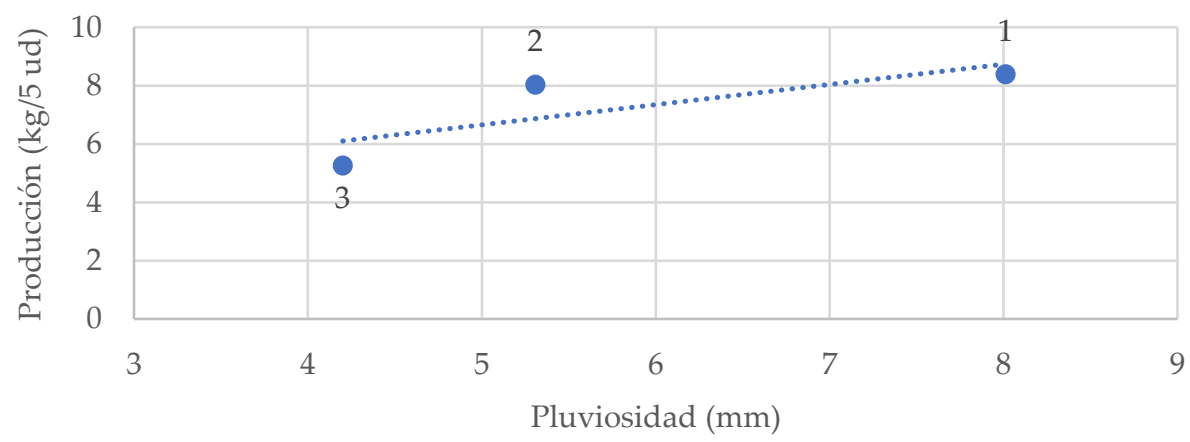

Figura 4. Relación entre la pluviosidad y la producción obtenida en cada uno de los marcos de muestreo. 


\section{Congreso Nacional de Riegos CARTAGENA 2021}

En cuanto a la comparación de la pluviosidad, producción y NDVI en los marcos de muestreo (Figuras 2, 3, y 4), se puede observar a través de una línea de tendencia que existe una relación directa entre el agua de riego aplicada (obtenida mediante la simulación) y el desarrollo del cultivo, teniendo en cuenta que cada uno de los marcos se encuentra en una zonificación de suelo diferente, donde por potencial productivo del suelo, el cultivo se desarrollará de distinta forma.

Para la caracterización del cultivo y la futura calibración en el programa AquaCrop, se realizó seguimiento fenológico con 19 muestreos, controlando además el correcto desarrollo del cultivo y sus diferente etapas fenológicas (en la escala $\mathrm{BBCH}$ ). Los mismos días que se realizaron los seguimientos fenológicos, se llevó a cabo un vuelo con un dron DJI Mini 2, para obtener imágenes RGB del cultivo y determinar así el grado de cobertura, obteniendo curva Kc del cultivo. Por lo que el siguiente paso es realizar la calibración del cultivo en AquaCrop y las simulaciones de cultivo correspondientes a cada sector de riego y zonificación por tipo de suelo, con el objetivo de obtener los kilogramos por metro cuadrado de parcela, obteniendo los índices; productividad del agua $\left(\mathrm{kg} / \mathrm{m}^{3}\right)$ y productividad de la energía $(\mathrm{kg} / \mathrm{kWh})$.

\section{Conclusiones}

El modelo integrado es capaz de simular un sector real de riego, teniendo en cuenta las variaciones de presión en cabecera, la no uniformidad de presiones en los diferentes aspersores y la dirección e intensidad del viento. Como prueba de que la simulación se ajusta a la realidad, se ha obtenido una evidente relación visual entre la pluviosidad en cada metro cuadrado con el índice NDVI, además, se ha conseguido relacionar directamente la pluviosidad con la producción de cebollas muestreadas en los diferentes puntos.

\section{Referencias}

1. FAO 2009. Cómo alimentar al mundo 2050. Foro de expertos de alto nivel: URL_http://www.fao.org/fileadmin/templates/wsfs/docs/synthesis_papers/C\%C3\%B3mo_alimentar_al_mundo_en_2050.pdf. Consultado: 20 de junio de 2021.

2. Moutonnet, P. Yield response factors of field crops to deficit irrigation. Food and Agriculture Organization of the United Nations (FAO). Deficit irrigation practices. 2002, pp. 11-15.

3. Carrillo-Cobo, M. T.; Camacho-Poyato, E.; Montesinos, P.; Rodríguez Díaz, J. Assessing the potential of solar energy in pressurized irrigation networks. The case of Bembézar MI irrigation district (Spain). Spanish journal of agricultural research 2014, volume $12 n^{\circ}$ 3, pp. 838-849.

4. Tarjuelo, J. M.; Rodriguez-Diaz, J. A.; Abadía, R.; Camacho, E.; Rocamora, C.; Moreno, M. A. Efficient water and energy use in irrigation modernization: Lessons from Spanish case studies. Agricultural Water Management 2015, volume 162, pp. 67-77.

5. Martínez Alzamora, F. EPANET. Una potente herramienta para el análisis de redes de distribución de agua a presión. Revista Hidropres. Tecnología de Captación, Gestión y Tratamiento Del Agua., 2003, pp. 30-37.

6. Rosman, L. A. Epanet 2 manual de usuario. US Environmental Protection Agency, Cincinnati, Ohio, 2001; pp. 1-2.

7. Montero, J.; Tarjuelo, J.; Carrión, P. SIRIAS: A simulation model for sprinkler irrigation. Irrigation Science, 2001, volume $20 n^{\underline{0}}$ 2, pp. 85-98.

8. Boote, K. J.; Jones, J. W.; Hoogenboom, G.; White, J. W. The role of crop systems simulation in agriculture and environment. International Journal of Agricultural and Environmental Information Systems (IJAEIS), 2010, volume $1 n^{\circ}$ 1, pp. 41-54.

9. Montoya Sevilla, F. Calibración y validación de modelos para la simulación de patata (Solanum tuberosum L.) bajo diferentes tratamientos de riego con pivot en condiciones semiáridas. Tesis doctoral, Universidad de Castilla-La Mancha, Albacete, 2013.

10. Steduto, P.; Hsiao, T. C.; Fereres, E.; Raes, D. Respuesta del rendimiento de los cultivos al agua. Estudio FAO: Riego y Drenaje (FAO), 2012, spa no 66, pp. 1-3.

11. Carrión, P.; Tarjuelo, J.; Montero, J. SIRIAS: a simulation model for sprinkler irrigation. Irrigation Science, 2001, volume $20 n^{\circ}$ 2, pp. 73-84. 